\title{
Multiple-scale object-oriented building extraction method from high resolution image
}

\section{Li Chaokui*, Fang Jun, Chen Jianhui, Zhou Qinglan and Zhou Qian}

${ }^{1}$ National-Local Joint Engineering Laboratory of Geo-Spatial Information Technology, Hunan University of Science and Technology, Xiangtan, Hunan, 411201, China.

${ }^{2}$ Hunan Province Engineering Laboratory of Geospatial Information, Hunan University of Science and Technology, Xiangtan, Hunan, 411201, China.

${ }^{3}$ Institute of Geospatial Information Science, Hunan University of Science and Technology, Xiangtan, Hunan, 411201, China.

Accepted 3 January, 2018

\begin{abstract}
Based on high-resolution remote sensing data and the e-Cognition Developer platform, in this paper, we make full use of rich spectral, spatial, texture and geometry information of high resolution QuickBird images in order to classify building areas successfully. The object-oriented multiple-scale segmentation method and the nearest neighborhood and membership function classification method are applied to classify the study area into five land categories; they are residential building, green space, road, leisure area and bare area respectively. On the basis above, the residential building information is extracted eventually. The experiment results show that: compared with the conventional pixel-by-pixel classification method, the object-oriented classification method proposed in this paper can effectively avoid the fragmentation of the segmented regions, which is more complete, accurate and efficient in land classification.
\end{abstract}

Keywords: Object-oriented, multiple scale segmentation, high resolution, residential buildings extraction.

*Corresponding author. E-mail: chkl_hn@163.com.

\section{INTRODUCTION}

With the rapid development of sensor technology and the increasing of the resolution of remote sensing images, it is the trend of taking advantages of high-resolution image in the remote sensing field in $21^{\text {st }}$ century ( $\left.\mathrm{Li}, 2003\right)$. Remote sensing images with high spatial resolution have rich spatial features, feature geometry and texture information processing. Traditionally, we process the images based on pixels, especially spectral statistics. And, it is hard to acquire good results (Peng et al., 2006). In order to identify and classify different categories from high resolution images efficiently, the authors take advantage of the object-oriented analysis technique based on high spatial resolution image characteristic as well as different image scale (Aplin et al., 1999). In order to perform such a classification, the authors employ the commercial software (Benz et al., 2004), which is widely used for multiple scale object-oriented segmentation on high resolution remote sensing images. This paper uses the object-oriented classification method to process multiple scale image segmentation and rule classification, to extract the information of residential buildings in the experimental area. Finally, the authors compare the results with traditional pixel-based classification method each other and analyse the significance of the objectoriented classification method in the rapid acquisition of the information in the high-resolution remote sensing image.

\section{OBJECT-ORIENTED CLASSIFICATION METHOD}

The object-oriented image classification method includes image segmentation, classification, post classification and accuracy assessment. Firstly, using the objectoriented method for processing the images with multiplescale segmentation, and the information should be described by a variety of scales (Cao et al., 2006). Then 
to establish the classification system, it should extract the surface feature information. Finally, classifier is used to extract many features of remote sensing images (Baatz and Schape, 1999).

\section{Remote sensing image segmentation}

Remote sensing image segmentation is to divide and refine the image into a plurality of interconnected image object area. It takes a collection of homogeneous pixels as a basic unit of image segmentation, so that it can avoid the isolated feature divided into adjacent heterogeneous pixels. After segmentation, the gray, color and texture of image objects in the same region are similar or identical (Benz, 2001; Zhu, 2003). For instance, the matching of acquired target category and segmentation objects is higher, the segmentation results are better. The primary criteria in the segmentation processing are the minimum of image heterogeneity. The heterogeneity standard includes spectra and shape. It is calculated from the initially integrated color and shape properties which are generated before image objects become complicated. Currently, the primary segmentation method contends region-based segmentation method, based on the edge of the image segmentation method, threshold-based segmentation method (Chang-bao and Ji-hong, 2006). The heterogeneity value (f) use shape heterogeneity $h_{\text {shape }}$ and spectral heterogeneity $h_{\text {colour }}$ to compact.

$f=h_{\text {shape }}+h_{\text {colour }}$

The shape heterogeneity $\left(h_{\text {shape }}\right)$ use compactness $\left(h_{\text {compact }}\right)$ and smoothness $\left(h_{\text {smooth }}\right)$ to compact.

$h_{\text {shape }}=w_{\text {compact }} \cdot h_{\text {compact }}+\left(1-w_{\text {compact }}\right) \cdot h_{\text {smooth }}$

Where $w_{\text {compact }}$ is the user defined weight for color with $0=\mathrm{w}=1$, the shape criterion consists in two parameters: the smoothness $\left(h_{\text {smooth }}\right)$ and the compactness $\left(h_{\text {compact }}\right)$ criterions.

$$
\begin{aligned}
& h_{\text {compact }}=n_{\text {merge }} \cdot \frac{l_{\text {merge }}}{\sqrt{n_{\text {merge }}}}-\left[n_{o b 1} \cdot \frac{l_{o b 1}}{\sqrt{n_{o b 1}}}+n_{o b 2} \cdot \frac{l_{o b 2}}{\sqrt{n_{o b 2}}}\right] \\
& h_{\text {smooth }}=n_{\text {merge }} \cdot \frac{l_{\text {merge }}}{b_{\text {merge }}}-\left(n_{o b 1} \cdot \frac{l_{o b 1}}{b_{o b 1}}+n_{o b 2} \cdot \frac{l_{o b 2}}{b_{o b 2}}\right)
\end{aligned}
$$

The spectral heterogeneity $\left(h_{\text {color }}\right)$ is measured by the size of the image object itself.

$h_{\text {color }}=\sum_{k=1}^{m} w_{k}=\left[n_{\text {merge }} \cdot \sigma_{\mathrm{k}}^{\text {merge }}-\left(n_{o b 1} \cdot \sigma_{\mathrm{k}}^{\mathrm{ob} 1}+n_{o b 2} \cdot \sigma_{\mathrm{k}}^{\mathrm{ob} 2}\right)\right]$

Where $n_{\text {merge }}$ is the combined object pixels, $k$ is the image layers, $w_{k}$ is the image layer weights, $\sigma_{\mathrm{k}}^{\text {merge }}$ is the combined spectral standard deviation, $n_{o b 1}$ is the pixels of object 1 before merging, $\sigma_{\mathrm{k}}^{\mathrm{ob} 1}$ is the spectral standard deviation of object 1 before merging, $n_{o b 2}$ is the pixels of object 1 before merging, $\sigma_{\mathrm{k}}^{\mathrm{ob} 2}$ is the spectral standard deviation of object 1 before merging.

\section{Characteristics of image objects}

The analysis of image objects characteristics is also an important content in the object-oriented classification method. In order to perform an effective classification, we should accurately analyse a variety of object information. The object-oriented characteristics mainly consist of image object characteristics (spectral, shape, texture, layer characteristics), the related characteristics between classes (adjacent image object relation, child object relation) (Hang, 2003; Qi, 2009) and global characteristics (with the whole classes and full view related characteristics, etc). Finally, we use these characteristics to construct a knowledge base system of various object hierarchies.

\section{EXPERIMENT AND RESULT ANALYSIS}

\section{Study area and data pre-processing}

In recent years, object-oriented image classification has been researched thoroughly in order to monitor land use changes successfully. Due to the different experimental region and purpose, it is so different on image segmentation and classification algorithms, and it has no universal method for all cases. We depend on concrete conditions to analysis and find out suitable classification 
method.

Taian is located in central of Taishan Mountain in Shandong Province. It is an ancient city, which is famous for its profound content of history and culture and magnificent nature scenery, to attract many tourists at home and abroad. So it is very important to accurately master the changes in the buildings. It also can offer the decision-making basis for development stratagem of urban planning and development strategy.

The study area is a residential area of a town in Taian. The paper selected Taian Quick-Bird panchromatic bands image and multiple-spectral bands image for the experiment. Panchromatic band in Figure $1 \mathrm{~b}$ shows on grayscale, but it has a wealth of spatial characteristics, such as shape, texture, and topological relation. Multiplespectral band image in Figure 1a by the uptake of multiple single-band radiation of feature multiple band spectral information has more bands and colorful information, but the spatial resolution is lower.

In order to improve the accuracy of the extracted information, we do the image previous process, including 4R3G2B false color composite, cutting and fusion. Select the open fusion dialog box Image Interpreter/Spatial Enhancement/Resolution Merge on the ERDAS icon panel tool bar, and set the reasonable parameters. Finally, a fusion image with a resolution of $0.61 \mathrm{~m}$ is obtained. The result of the fusion is shown in Figure 1c, which has both high spatial resolution of panchromatic band and spectral information of the multiple-spectral bands.

\section{Feature analysis of study area}

Building type extracted from this paper is mainly the architecture residential buildings. The study area is made of rule distribution classes, such as residential building, grassland, roads, bare land, and leisure area. There are some facts that different objects have the same spectrum, because the diversity of the shape of residential buildings and roof material. The paper focused on the study area of residential buildings. Residential buildings in the study area have the following distribution characteristics:

1. Spectral characteristics: In Figure 1c, the image has rice color because of the diversity of roof material and the influence of building shades. Therefore, the spectral features will be changed based on the different roof material.

2. Spatial characteristics: Texture information explains the detailed structure of the target objects or objects internal components. The spatial resolution of Quick-Bird Image is up to $0.61 \mathrm{~m}$, and it has rich texture information. From it, we can see more details of the feature morphological characteristics. Residential buildings in the study area have regularly geometric distribution space, and its outline is clear, obvious and rich edge information.
The shape of residential buildings in the study area is regularly rectangular distribution and showing a combination of regular arrangement.

\section{Image segmentation of study area}

In e-Cognition Developer, the parameter combination is depended on the characteristics of different feature types from data and images to determine the best segmentation parameters. The scale parameter represents the standard colors of objects. It is the important standard to generate image objects. In segmentation, the best segmentation parameter will be determined after several attempts and analysis (Table 1). The segmentation scale (Figure 2) is 16 . The color factor is 0.7. After segmentation, irregular polygon of image objects is larger. Various classes are complete after segmentation, such as residential building.

\section{Object-oriented classification}

This paper is based on the e-Cognition Developer Platform. It is an image processing and analysis software with object-oriented, multiple scale analysis and fuzzy rules. It takes the nearest and membership function to classify images. Nearest neighbor (Nearest Neighbor) classification needs to define sample and feature space for each class. The feature space can combine any characteristics. At the beginning, we select fewer samples, so that we can add or remove samples to improve the effect in the subsequent processing. Nearest neighbor classification can evaluate the correlation of the object features, and is easy to handle the overlap of multiple-dimensional feature space. And it is also easy to quickly deal with the relationship in the class hierarchy. Membership function is a method to express the value between 0 and 1 , that is the membership value $a \in[0 \ldots$ 1]. Through combined logic operators, we calculate the range of classes and define precisely the standard of the object classes. It can make use of relevant characteristics between classes and object characteristics, and is easy to edit and adjust every feature. Also it provides a very transparent relationship between the feature values and the membership of classes.

In the process of building information extraction, the hierarchy is created in e-Cognition through the classification feature information. We take the nearest neighbor and membership function method to extract building features for reaching the classification of residential buildings. The rules of classification of membership function method are ration when resultmerge Boolean value is greater than [0.21, 0.3535, 0.497], brightness $>50$ and standard deviation $>2$. Figures 3 and 4 are the result of the nearest neighbor and the membership function classification.

From the processing of whole classification, the key is 


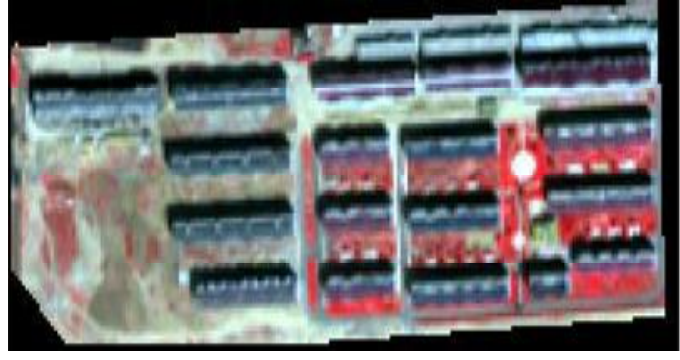

a

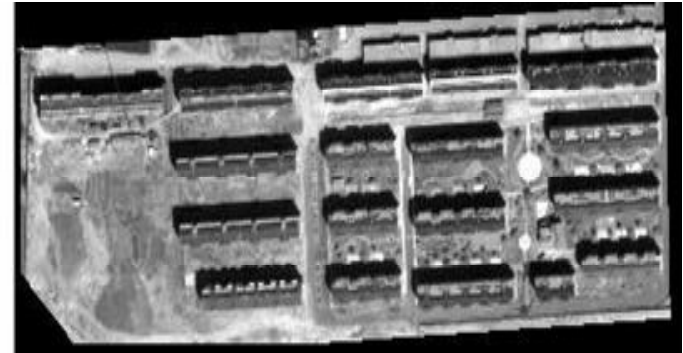

b

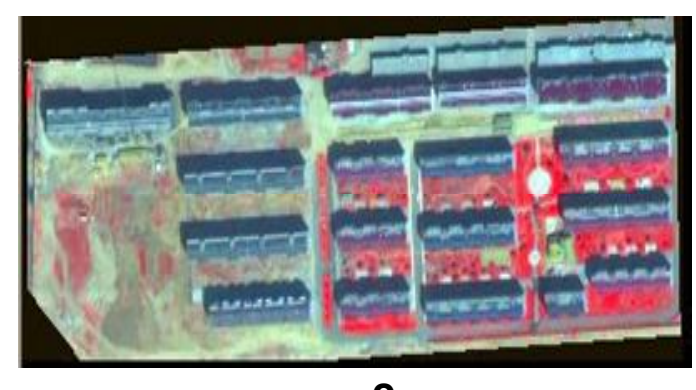

C

Figure 1. The image preprocessing results. (a) Multiple-spectral bands cutting image; (b) Panchromatic bands cutting image; (c) The fusion result of multiple-spectral bands and panchromatic bands.

Table 1. Different scale parameters of image features.

\begin{tabular}{cl}
\hline Scale parameters & The description of the classification result characteristics \\
\hline 10 & $\begin{array}{l}\text { Because the scale of the segmentation is small, the image objects established is } \\
\text { smaller. Residential buildings, grassland, roads, bare land is too broken. It is not } \\
\text { beneficial to the integrity of the extraction of residential building information. }\end{array}$ \\
15 & $\begin{array}{l}\text { All classes of residential buildings, grassland, roads, and bare land image objects are } \\
\text { made of large irregular polygons. But the texture is not irregular, and the objects are } \\
\text { quite broken. }\end{array}$ \\
16 & $\begin{array}{l}\text { Irregular polygon area of image objects change larger. Residential buildings and } \\
\text { grassland can be extracted. The integrity of bare land, grassland, roads and residential } \\
\text { buildings is enhanced. }\end{array}$ \\
& $\begin{array}{l}\text { Irregular polygon area of image objects change much larger. Residential buildings and } \\
\text { grassland can be extracted. But the integrity of a residential building is bad. }\end{array}$ \\
20 & $\begin{array}{l}\text { Irregular polygon area of image objects change much larger. The integrity of bare land } \\
\text { and grassland is enhanced. But the integrity of some residential buildings is bad. }\end{array}$ \\
30 & $\begin{array}{l}\text { Irregular polygon area of image objects change much larger. The integrity of grassland } \\
\text { is well. But part of residential buildings and roads is mixed and cannot be divided. }\end{array}$ \\
\hline
\end{tabular}

image segmentation and object characteristics. If the segmentation scale is suitable, the segmentation features is similar to the object information, and the result is meaningful. It is a hard work to select suitable characteristics from the numerous characteristics. Judging from the final classification result, the classification is quite accurate.

\section{Result and analysis}

The object-oriented method was applied to segmentation, 


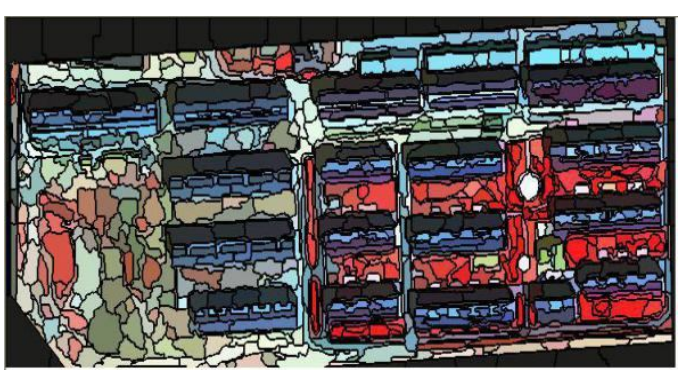

Figure 2. Parameters of study area: $16,0.3,0.5$.

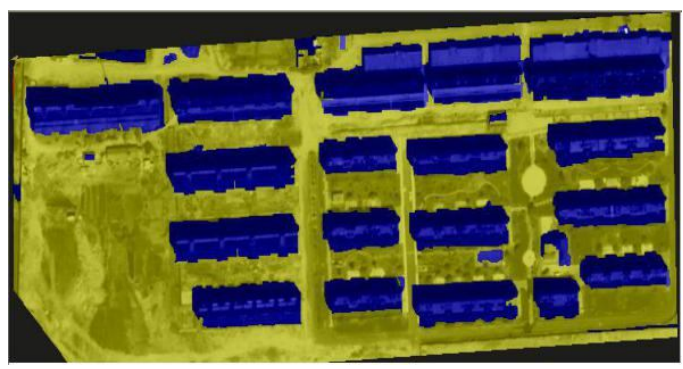

Figure 3. Classification of nearest neighbor.

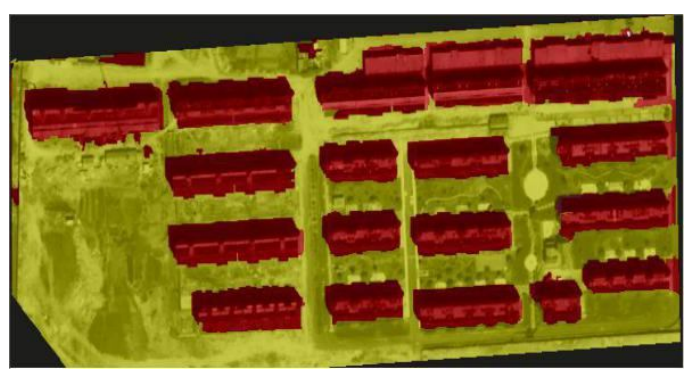

Figure 4. Classification of membership function.

nearest neighbor and membership function for study area. The study area is divided into five classes, such as residential buildings, grassland, roads, leisure area and bare land, to extract residential buildings information. Finally, we use probability of the best classification results and the stability of classification to do precision evaluation. Classification stability is calculated in the best value and the second best value. The best classification result is calculated from assigned image objects to a class. The maximum value of classification results in the five classes is 1 . The mean value is greater than 0.92 . The standard deviation is below 0.1027 . The mean value and the stability of classification is less than 0.2882 . The result of the classification is good.

From the comparative of the two extracted methods, Figures 3 and 4 are more complete. Figures 5 and 6 are more broken, and appear "salt and pepper" effects. The information redundancy is high. Because the traditional pixel-based classification method only uses image

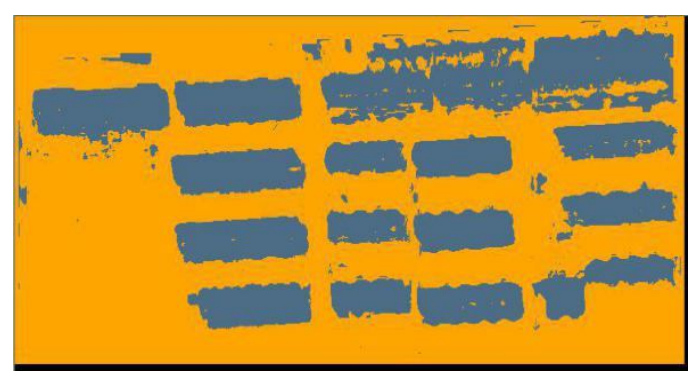

Figure 5. Supervised classification.

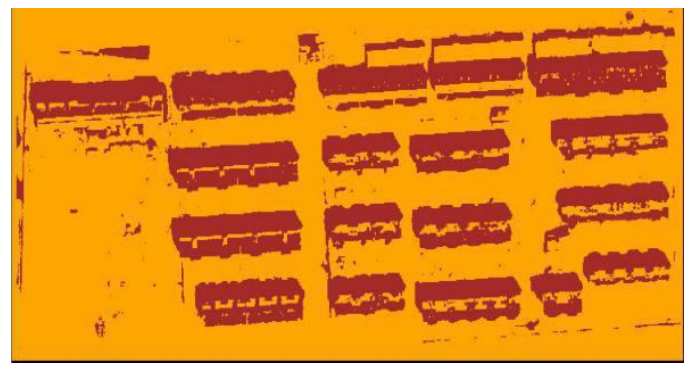

Figure 6. Unsupervised classification.

spectrum information, the residential buildings information extracted is made of discrete pixels, and cannot describe the contour and shape information of residential buildings. It easily leads to the phenomenon that different objects have the same spectrum. However, the object-oriented method not only uses spectral information, but also makes full use of the rich spatial information of high resolution image. It is more complete to achieve residential buildings information.

\section{CONCLUSION}

Based on object oriented image classification method, multi-scale segmentation technology is adopted, and nearest neighbor classification and membership function classification are applied to extract the building information in the experimental area. The problem of the scale of image segmentation and the selection of its parameters are studied. The results of this method and the traditional supervised classification method based on pixel are compared with each other. The segmentation results are obtained by continuous transformation of the segmentation scale, and the segmentation results are compared each other. Compared the segmentation results, the optimal segmentation scale is determined. The main research conclusions are as follows:

1. The classification method of object-oriented remote sensing image has many advantages, such as high precision, convenient and quick. The object oriented remote sensing image analysis method not only takes 
advantage of the inherent spectral characteristics of the image itself, but also fully considers the shape, texture and hierarchy of the objects in the image.

2. Object oriented classification method adopts multiplescale segmentation technology. It can divide the image into a number of object polygons, and the setting of segmentation parameters will directly affect the quality of segmentation. In the experiment, we use continuous transformation scale to get different segmentation results, and analyze the accuracy of surface classification under different scales, and get better classification information, so as to determine the optimal segmentation scale.

3. E-Cognition supports two different classification methods, which are the most adjacent classification and the membership function classification. In the experiment, the two methods are used to classify the sample area and the building information of the sample area is extracted. Developing a knowledge base for specific taxonomy means editing and refining the class description of each class. In the processing of multidimensional feature space, the nearest neighbor classification method is more suitable than the membership function method.

4. Compared with the pixel based supervised classification method, we can see clearly that the object oriented classification method has higher classification accuracy and is more accurate and efficient in building information extraction. It can effectively avoid the "Pepper phenomenon", clearly distinguish the "synonyms spectrum" and "foreign body in the same spectrum" phenomenon, it has great advantage in the high resolution image classification.

\section{ACKNOWLEGEMENT}

This paper is funded jointly by the projects: Nature Science Funding number: 41571374, the Key Laboratory Open Funding Project of Hunan Province (No: JL16K01, CT16K02), the Key Research Project of Hunan Education Ministry (No: 16A070), Nature Science Joint Funding of Hunan Province and Xiangtan Local (No: 2017JJ4037).

\section{REFERENCES}

Aplin P., Atkinson P. M., Curran P. J. (1999). Per-field classification of land use using the forthcoming very fine spatial resolution satellite sensors: problems and potential solutions. In Advances in Remote Sensing and GIS Analysis. pp: 219-239.

Baatz M., Schape A. (1999). Object-oriented and multi-scale image analysis in semantic networks. In: Proc of the 2nd International Symposium on Operationalization of Remote Sensing. August 16 20th. Ensehede ITC.

Benz U. (2001). Definiens Imaging GmbH: Object-Oriented Classification and Feature Detection. IEEE Geoscience and Remote Sensing Society Newsletter, (September), 16-20.
Benz U. C., Hofmann P., Willhauck G., Lingenfelder I., Heynen M. (2004). Multi-resolution, object-oriented fuzzy analysis of remote sensing data for GIS-ready information. ISPRS J Photogrammetry Remote Sens, 58(3-4): 239-258.

Cao B., Qin Q., Ma H. (2006). Application of object-oriented approach to SPOT5 image classification: A case study in Haidian District, Beijing City. Geograp Geo-Informat Sci, 22(2): 46-49.

Chang-bao Y., Ji-hong D. (2006). Study of object-oriented based remote sensing image classification. J Jilin Univ, 36(4): 642-646.

Hang H. (2003). Scale Issues in Object-oriented Image Analysis [D]: [PhD dissertation]. Beijing: Institute of Remote Sensing Applications Chinese Academy of Sciences.

Li D. (2003). Towards the development of remote sensing and GIS in the 21st century. J Geomatics Informat Sci, 28(2): 127-131.

Peng G., Xia L., Bing X. (2006). Interpretation theory and application method development for information extraction from high resolution remotely sensed data. J Remote Sens, 10(1): 1-5.

Qi Y. (2009). Feature Extraction and Scale Effect Analysis of Highresolution Image Using Object-oriented Approach [D]: Master Dissertation]. Changchun: Northeast Normal University.

Zhu G. (2003). Remote sensing image analysis based on hierarchical multi-resolution structures. Geomatics and Information Science of Wuhan University, 28(3): 315-320.
Citation: Chaokui L., Jun F., Jianhui C., Qian Z (2018). M ultiple-scale object-oriented building extraction method from high resolution image. Afr J Eng Res, 6(1): 1-6. 